\title{
层状液晶中 $\mathrm{KCl}$ 超微粒子的制备*
}

严鹏权 㭏 荣沈 明 朱霞石

(扬州大学师范学院化学系, 扬州 225002)

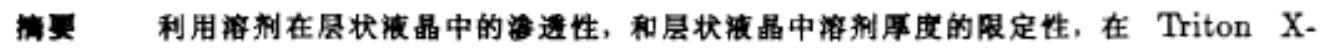

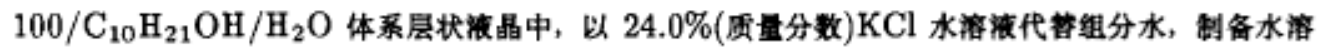

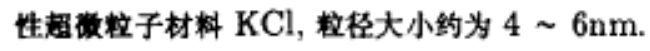

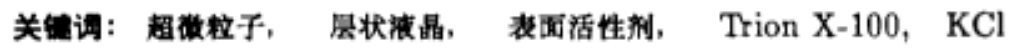

超微精子是极有应用价值的新型材料 ${ }^{[1-5]}$, 其大小通常在 $100 \mathrm{~nm}$ 以内, 有物理和化 学两种制备方法. 在化学法制备中, 主要依靠由表面活性剂生成的分子有序组合体(如胶 束, 微乳液等) 的大小限制了粒子生成的大小 ${ }^{[6]}$.

对非水溶性超微粒子材料的制备研究已有一定深度, 而水溶性超微粒子材料的制备一 直难以解决, 尽管水溶性超䵊粒子材料在实际工作中有着极重要的应用价值. 我们曾利用 分子有序组合体的另一种形式, 即层状液晶为介质, 制备了水楁性超微粒子 $\left[\mathrm{Co}\left(\mathrm{NH}_{3}\right)_{6}\right] \mathrm{Cl}_{3}$ 和 $\mathrm{CuSO}_{4} \cdot 5 \mathrm{H}_{2} \mathrm{O}^{[7,8]}$, 本文以 Triton $\mathrm{X}-100 / \mathrm{C}_{10} \mathrm{H}_{21} \mathrm{OH} / \mathrm{H}_{2} \mathrm{O}$ 体系层状夜晶为介质, 制备了 水嚓性超微粒子枋料 $\mathrm{KCl}$.

\section{1 实验部分}

武剂与仪器: Triton X-100 (Aldrich 公司, 分析纯). 正癸醇 (分析纯), 水为二次蒸 馏水, 无水乙醇 (分析纯) $\mathrm{KCl}$ (分析纯) $\mathrm{X}$ 射线衍射仪 (日本理学公司), 电子显微镜 (日立 $600 \mathrm{~A}-2$ ), 超声波清洗器 (上海超声波仪器厂).

层状液晶单相区减的确定: 按不同组成配样, 充分振荡混合后, 置恒温水浴 $(20 \pm 0.1$ ${ }^{\circ} \mathrm{C}$ ) 中狟温 12 小时以上, 由偏振光显微镜 (上海光学仪器厂 $59 \mathrm{X}$ 型) 判断为何种滨致液晶 单相.

小角 X 射线衍射测量: 将试样按比例放入有盖试管中, 混合均匀后离心 20 分钟 (转 速为 $5000 \mathrm{r} \cdot \mathrm{min}^{-1}$ ) 以取赶气泡, 然后吸入专用毛细管 (直径为 $0.7 \mathrm{~mm}$ ) 中, 用石腊密封两 端后即可进行测定, 仪器为 $\mathrm{D} / \mathrm{max}-\mathrm{rc}$ 型转耙 $\mathrm{X}$ 射线衍射仪 (日本理学公司), 测定过程中 使用了 $\mathrm{Ni}$ 滤波片， $\mathrm{Cu}$ 阳极靯 $(\lambda=0.1542 \mathrm{~nm})$, 管压为 $50 \mathrm{kV}$, 管流为 $180 \mathrm{~mA}$.

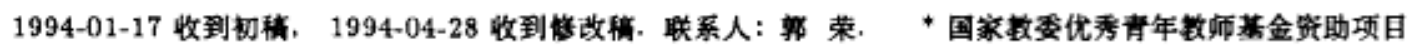


$\mathrm{KCl}$ 超微粒子的制备：以 $24.0 \%$ (质量分数, 下同) $\mathrm{KCl}$ 水溶液代替组分水制备层状液 晶. 由于嚓剂在层状液晶两亲双层中的渗透, 降低了 $\mathrm{KCl}$ 的溶解度, 使 $\mathrm{KCl}$ 结晶析出, 在高速离心机上离心分离后, 再用无水乙酷充分洗涤, 在超声波清洗器中振荡分散.

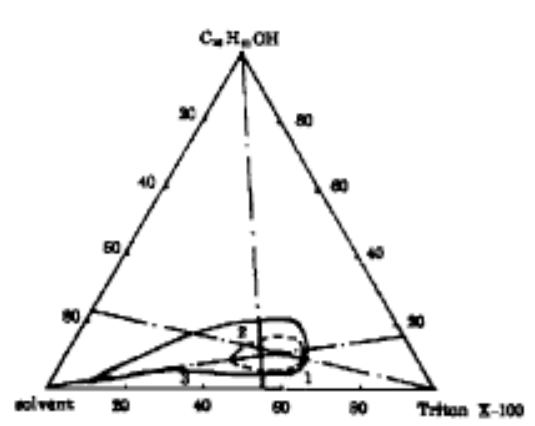

图 1 Triton $\mathrm{X}-100 / \mathrm{C}_{10} \mathrm{H}_{21} \mathrm{OH}$ $/ \mathrm{H}_{2} \mathrm{O}$ 体乗部分相园

Fig.1 A partial diagram of

Triton $\mathrm{X}-100 / \mathrm{C}_{10} \mathrm{H}_{21} \mathrm{OH}$

$/ \mathrm{H}_{2} \mathrm{O}$ system

Solvent: $--\mathrm{H}_{2} \mathrm{O}$;

- . - $24.0 \% \mathrm{KCl}$ (aq.)
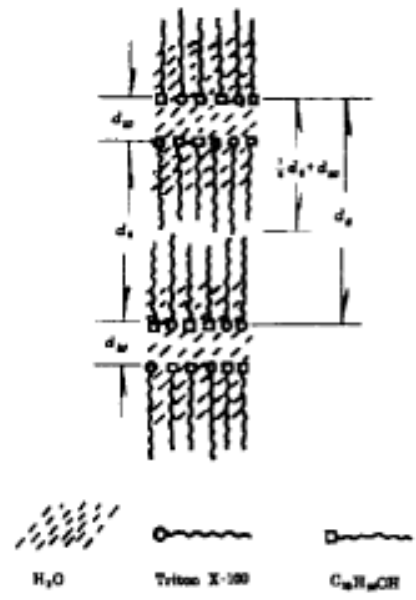

图 2 屌状流畠结构示来国

Fig.2 Illustration of lamellar

liquid crystal

$d_{e}-$ interlayer spacing

$d_{0}$ - thickness of amphoteric

double layer

$d_{w}-$ thickness of solvent layer

\section{2 结果与讨论}

\section{1 层状液晶区域与结构特征}

图 1 中实线所示的单相区域为 Triton X-100/ $\mathrm{C}_{10} \mathrm{H}_{21} \mathrm{OH} / \mathrm{H}_{2} \mathrm{O}$ 体系层状液晶单相区 城. 图 2 则表示了层状液晶的结构示意图. 通过小角 $\mathrm{X}$ 射线测量 ${ }^{[9-13]}$, 可得到不同重量比 $\mathrm{C}_{10} \mathrm{H}_{21} \mathrm{OH} /$ Triton $\mathrm{X}-100$ 条件下, 该层状液晶的有关结构参数 $d_{0}$ 和溶剂渗透率 $p($ 表 1$)$ 以 及层状液昆楁剂层军度 $d_{w}$ 与含水量 $\phi$ 之间的关系 (图 3). 这些结果表明, 楁剂的渗透性 增强, 两亲及层内的楁剂量增加, 存在于溶剂层内的渒剂量减少, $d_{w}$ 值减小, 反之亦然.

由图 3 和表 1 数搭还可见, 本研究所选层状液晶体系中溶剂的渗透率可高达 $52 \sim 55 \%$, 而 溶剂层厚度一般小于 $3 \mathrm{~nm}$, 这些数括表明在该层状液晶中制备本楁性超微精子是可能的.

\section{2 层状液晶中 $\mathrm{KCl}$ 超微粒子的制备}

\section{2 .1 层状液晶对 $\mathrm{KCl}$ 表观溶解度的影响}

在图 1 所示 Triton X-100/ $\mathrm{C}_{10} \mathrm{H}_{21} \mathrm{OH} / \mathrm{H}_{2} \mathrm{O}$ 体系层状液晶区城内取点, 以一系列不同 浓度的 $\mathrm{KCl}$ 水溶液代替组分水, 制备层状液晶, 以偏振光显德镜观察是否有粒子析出, 以 确定刚开始有粒子析出时的 $\mathrm{KCl}$ 水溶液的浓度, 此浓度即为在该组成层状液晶体系中 $\mathrm{KCl}$ 的表观溶解度. 图 4 则表示了不同重量比 $\mathrm{C}_{10} \mathrm{H}_{21} \mathrm{OH} /$ Triton X-100下, 层状液晶中含水 量对 $\mathrm{KCl}$ 表观嚓解度的影响. 在 $20{ }^{\circ} \mathrm{C}$ 时 $\mathrm{KCl}$ 在水中的溶解度为 34.0 克 $/ 100$ 克溶剂, 由 
图 4 可见在层状液晶中 $\mathrm{KCl}$ 的表观詹解度降低, 并且随层状夜晶中含水量的增加, 其影 响减弱, $\mathrm{KCl}$ 表观察解度增加. 其机理可能是在含 $\mathrm{KCl}$ 的层状液晶中, $\mathrm{KCl}$ 存在于嚓剂 层内, 由于溶剂向两亲双层内渗透, 使得楁剂层内的 $\mathrm{KCl}$ 溶液过饱和, 因而析出. 随含水

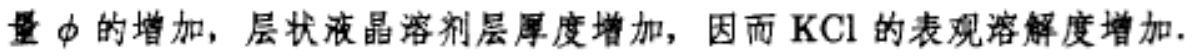

\section{表 1 层状洨盢的结构少数}

Table 1 The structure parameters of lamellar liquid crystal

\begin{tabular}{lcc}
\hline $\mathrm{C}_{10} \mathrm{H}_{21} \mathrm{OH} /$ Triton $\mathrm{X}-100$ & $d_{0} / \mathrm{nm}$ & $p$ \\
$w / w$ & & \\
\hline $17 / 83$ & 3.60 & 0.527 \\
$23 / 77$ & 3.55 & 0.547 \\
\hline
\end{tabular}

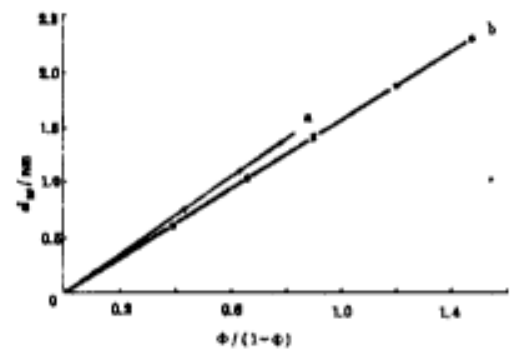

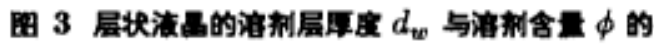
关聚

Fig.3 The relation between the solvent thickness of liquid crystal $d_{w}$ and solvent content $\phi$ $\mathrm{C}_{10} \mathrm{H}_{21} \mathrm{OH} /$ Triton $\mathrm{X}-100(w / w)$ :

a) $17 / 83$; b) $23 / 77$
$2.2 .2 \mathrm{KCl}$ 水溶液 $(24.0 \%)$ 对层状液晶区城 的影响

图 1 中虚线表示了浓度为 $24.0 \%$ 的 $\mathrm{KCl}$ 水嚓洨对 Triton $\mathrm{X}-100 / \mathrm{C}_{10} \mathrm{H}_{21} \mathrm{OH} / \mathrm{H}_{2} \mathrm{O}$ 体系层状夜晶区域的影响, 由图可见, $\mathrm{KCl}$ 的存在降低了层状液晶的稳定性, 使得 Triton $\mathrm{X}-100 / \mathrm{C}_{10} \mathrm{H}_{21} \mathrm{OH} / \mathrm{H}_{2} \mathrm{O}$ 体系层状夜昆区 域缩小.

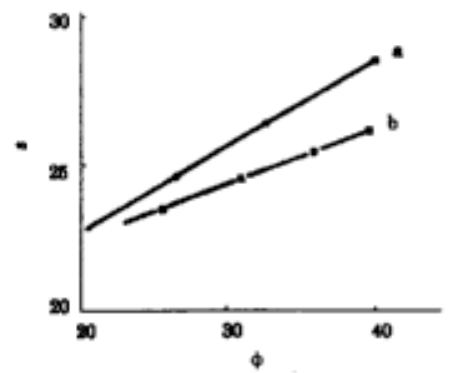

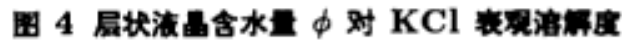
的影响

Fig.4 The effects of water content in the lamellar liquid crystal on the solubility of $\mathrm{KCl}$ $\mathrm{C}_{10} \mathrm{H}_{21} \mathrm{OH} /$ Triton $\mathrm{X}-100(w / w)$ :
a) $18 / 82$; b) $15 / 85$

\subsection{3 层状液晶中 $\mathrm{KCl}$ 超微粒子的制备及其表征}

按图 1 中所示样品 $1 \sim 3$ 的组成配样, 即有 $\mathrm{KCl}$ 粒子析出. 经高速离心机分离出粒 子, 用无水乙醇充分洗溙, 再经振荡分散, 即得 $\mathrm{KCl}$ 超德粒子. 样品 $1 \sim 3$ 经 TEM 测定, 它们的微粒大小分别约为 $4 、 4 、 6 \mathrm{~nm}$, 样品 1 处制得的 $\mathrm{KCl}$ 粒子, 分布比较均勺.

图 5 表示了图 1 中样品 1 的 X 射线图谱, 显然所得的 $\mathrm{KCl}$ 样品的 X 射线图谱与标样 完全一致, 说明所制 $\mathrm{KCl}$ 超微粒子结构与纯度的可靠性.

比较 TEM 测定结果与图 3 的结果可见, 以层状液晶为介质所制得的 $\mathrm{KCl}$ 超微粒子 的大小与层状液晶的溶剂层屏度有关. 图 3 已表明层状液昆楁剂层厚度一般小于 $3 \mathrm{~nm}$. 显 然, 析出的 $\mathrm{KCl}$ 粒子的大小受到了层状夜晶溶剂 (水) 层厚度的限制. 


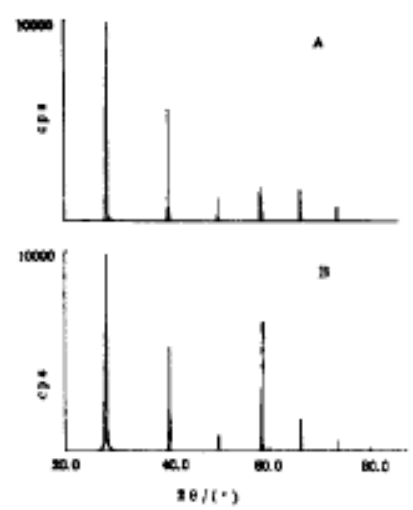

国 $5 \mathrm{X}$ 柇找街射图漕

Fig.5 X-ray spectrum
A) standard sample
B) sample made in the liquid crystal

\subsection{4 层状液晶各组分含量对 $\mathrm{KCl}$ 超微粒. 子制备的影响}

结合图 1 和 TEM 测定结果可见, 无论 在确定重量比 $\mathrm{C}_{10} \mathrm{H}_{21} \mathrm{OH} /$ Triton X-100 下增 加含水量 (比较样品 1 和 3 ), 还是在确定重 量比 $24.0 \% \mathrm{KCl}$ 水溶液/Triton X-100 下改 变 $\mathrm{C}_{10} \mathrm{H}_{21} \mathrm{OH}$ 含量 (比较样品 3 和 2 ) 或确 定重量比 $\mathrm{C}_{10} \mathrm{H}_{21} \mathrm{OH} / 24.0 \% \mathrm{KCl}$ 溶液下改 变 Triton X-100 含量 (比较样品 2 和 1), 所 得 $\mathrm{KCl}$ 超微粒子大小无显著变化. 这些结 果表明, 在 Triton X- $100 / \mathrm{C}_{10} \mathrm{H}_{21} \mathrm{OH} / \mathrm{H}_{2} \mathrm{O}$ 体 系层状液晶中改变三组分含量对 $\mathrm{KCl}$ 越微 粒子的制备均无显著影响. 其原因可能与 层状波晶水层厚度随组分变化很小有关。

由图 3 所示结果可见, 在确定含水量下, 如果改变 $\mathrm{C}_{10} \mathrm{H}_{21} \mathrm{OH} /$ Triton X-100 的重量比, 从 $23 / 77 \sim 17 / 83$, 层状液晶水层厚度只能改变 $0.1 \sim 0.2 \mathrm{~nm}$. 若在 $\phi /(1-\phi)=0.4 \sim 1.00$ 范围内 变化含水量, 层状液晶的水层厚度只能增加 $1 \mathrm{~nm}$, 如此小的变化不能对所析出的 $\mathrm{KCl}$ 超微 粒子的大小产生显著的影响.

\section{考文暆}

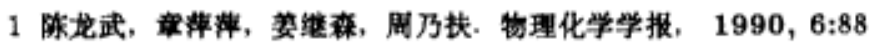

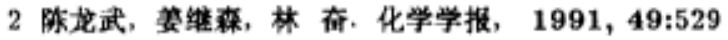

3 Matijevic E. Acc. Chem. Rec., 1981, 14:20

4 扬为忠夫。表面 (日), 1984, 22:177

5 张玉亭, 王寅生, 周乃扶等. 科学通报, 1985, 30:1160

6 Kanlovietal K. J. Colloid Interface Sci., 1987, 122:78

7 郭荣, 严影权, 刘正络, 沈明. 化学学报, 1994, 52:729

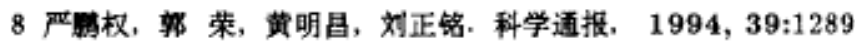

9 垶 荣, 物理化学学报, 1991, 7:(6): 703

10 Friberg S E, Guo Rong, Ward A J I. J. Phys. Chem., 1988, 92:7247

11 整荣, Friberg S E. 生物化学与生物物理学报, 1991, 23:89

12 熟荣. 槀等学校化学学报, 1991, 12:1526

13 Friberg S E, Guo Rong. Langmuir, 1988, 4:796 
The Preparation of Water-soluble Fine Particles $\mathrm{KCl}$ in the Triton $\mathrm{X}-100$ $/ \mathrm{C}_{10} \mathrm{H}_{21} \mathrm{OH} / \mathrm{H}_{2} \mathrm{O}$ Lamellar Liquid Crystal

Yan Pengquan Guo Rong Shen Ming Zhu Xiashi

( Department of Chemistry, Teacher's College, Yangzhou University, Yangzhou 225002)

\begin{abstract}
Water-soluble fine $\mathrm{KCl}$ particle was prepared by replacing component water with $24.0 \% \mathrm{KCl}$ aqueous solution in the Triton $\mathrm{X}-100 / \mathrm{C}_{10} \mathrm{H}_{21} \mathrm{OH} / \mathrm{H}_{2} \mathrm{O}$ lamellar liquid crystal. The preparation was based on the penetration of solvent in the lamellar liquid crystal and the limitation of the change of thickness of solvation layer in the lamellar liquid crystal. The size of water-soluble fine particle $\mathrm{KCl}$ was about $4-6 \mathrm{~nm}$.
\end{abstract}

Keywords: Fine particles, Lamellar liquid crystal, Surfactant, Triton X-100, $\mathrm{KCl}$ 